\title{
Radioactive Hot-spot Detection Using Unmanned Aerial Vehicle Surveillance
}

\author{
Yoeri Brouwer, Alberto Vale ${ }^{\star}$, Duarte Macedo, Bruno Gonçalves, Horácio Fernandes \\ * Instituto Superior Técnico \\ avale@ipfn.tecnico.ulisboa.pt
}

\begin{abstract}
This work proposes a solution to identify the number of sources of radiation, as well as their respective intensities and locations based on data acquired by Global Positioning System (GPS) receivers and affordable radiological sensors, such as Geiger-Müller counters (GMC). An optimization algorithm is required to minimize the estimation error in terms of location, intensity and number of sources of radiation given all the intensity measurements acquired in different locations, taking into account the sensors' models, background radiation intensity values and noise. Experimental results were achieved in a laboratory with controlled sources of radiation. The solution was also tested with real data gathered by a GMC connected to a mobile phone with a software application developed by the authors to synchronize the sensor readings with GPS data. The sensor and the mobile phone are attached to a quadcopter flying over the scenario with sources of radiation.
\end{abstract}

Keywords-UAV, Radioactive Source, Radioactivity, Hotspot Detection, Collimator.

\section{INTRODUCTION}

$\mathbf{T}$ HE defence against Chemical, Biological, Radiological and Nuclear (CBRN) threats is an increasing demand due to wars, terrorist attacks, disasters, or simply due to negligence. Recent projects have achieved improvements, but we are still far from safeguarding humankind against CBRN threats. NATO already has a Task Force working towards this objective and the National Academy of Engineering presented in 2008 "The 14 Grand Challenges for Engineering", where the prevention of nuclear terrorism was identified as one of the great challenges for the 21st century [1]-[3].

Radiological and nuclear threats are associated with radioactive elements that release large amounts of energy through alpha particles, beta particles, X-rays, gamma rays, and neutrons. This paper is particularly focused on these threats, which arise due to the emerging applications of nuclear technologies in industry, power production, medicine, and the military. It is also important to address radiological threats given the catastrophic consequences of nuclear bombs of the Second World War and the nuclear incidents which occurred in Chernobyl in 1986 and in Fukushima in 2011. In future work, the proposed approaches will be extended to chemical and biological threats.

Radiological and nuclear threats are often solely associated with nuclear bombs and nuclear fission power plants. However,

Y. Brouwer is with the Department of Electrical and Computer Engineering, Instituto Superior Técnico, Lisbon, Portugal, e-mail: yoeri.brouwer@ipfn.tecnico.ulisboa.pt.

A. Vale, B. Gonçalves and H. Fernandes are with the Instituto de Plasmas e Fusão Nuclear, Instituto Superior Técnico, Lisbon, Portugal.

D. Macedo is with the Department of Physics, Instituto Superior Técnico, Lisbon, Portugal.

Manuscript received May 13, 2019; revised [month day], 2019. apart from terrorist threats, nuclear materials can be commonly found in nuclear medicine waste products (e.g., radiation therapy for cancer treatment), abandoned mine sites, or even decommissioned radioactive material storage facilities.

As mentioned above, there are a number of projects which aim to address the issues of radiological and nuclear threats. MANUELA [4] is a project of the AREVA company, currently ORANO, a French multinational group in nuclear and renewable energy, where the mobile robot is equipped with LIDAR systems and it performs a 3D reconstruction of internal scenarios with the indication of the radiation levels of hotspots. Project REWARD [5] extends the radiological survey to large outdoor environments using radiological sensors installed on cars equipped with GPS receivers. The used sensors are multiple miniaturized solid state radiological sensors: Cadmium, Zinc and Tellurium (CZT) for gamma radiation detection and a high efficiency neutron detector based on new semiconductor technologies. SEAGULL [6] is a project based on Unmanned Aerial Vehicles (UAV) for surveillance and monitoring in the marine environment using video and thermal cameras. RIANA [7], also from AREVA, is a project with wheeled mobile robots with the capacity to operate in nuclear scenarios to provide radiological readings and take physical samples from hazardous scenarios. GAMMAex [8] is a project which uses UAVs to perform monitoring and recognition of chemical and radiological patterns in scenarios where biological, chemical, and radiological threats are identified.

The application of UAVs has been extended to scenarios where the danger of explosive atmospheres exists, through the construction of an ATEX (ABS Explosive) system. This project was mainly focused on the sensory part, being open to the application of new AI techniques. The ENCIRCLE project [9], funded by the European Union (EU), aims to strengthen the European CBRNe (CBRN and Explosive Materials) industry and its capabilities.

The use of Unmanned Aerial Vehicles (UAVs) enables further expansion of the total area coverage for a given scenario. Systems comprised of UAVs mounted with radiological sensors have already been tested as a solution to cover broader areas [10], [11], and commercial solutions already exist, such as [12] and [13]. Preliminary tests were performed by the authors of this paper applying Simultaneous Localization and Mapping (SLAM) techniques, commonly used in robotics, resulting in a $3 \mathrm{D}$ map of a scenario with radiological information [14]. This paper addresses the problem of estimating the quantity, intensity, and location of different sources of radiation based on observations and sensor models.

The rest of this paper is organized as follows. Section II 
introduces the variables used and describes the behaviour of the radiological sources. Section III describes the approaches which were studied in order to address the given problem, as well as the approaches which were selected for testing. Section IV illustrates and analyses the results obtained via simulation and real world experiments. Finally, section V summarizes the main conclusions of the work done.

\section{Problem Statement}

The problem is illustrated in Figure 1. The scenario may contain a number of radiological sources $i=0,1, \ldots, n$, represented by the symbol $s_{i}$. Each source has a position $\mathbf{p}_{s_{i}}=\left(x_{s_{i}}, y_{s_{i}}, z_{s_{i}}\right)$ and an activity $a_{s_{i}}$ representing the average number of particles emitted per second. The following four assumptions are made: first, the radiological sources are isotropic point sources; second, radiation intensity measurements are independent random variables each following a Poisson distribution; third, all the measurements have an equal exposure time; and fourth, the activity of each source remains constant over time, i.e., the duration of any measurement session is negligible compared to the half-life of the sources.

A set of observations are made in this scenario, represented by the symbol $o_{j}, j=1,2, \ldots, m$. Similarly, each observation has a position $\mathbf{q}_{o_{j}}=\left(u_{o_{j}}, v_{o_{j}}, w_{o_{j}}\right)$ and a measured intensity $c_{o_{j}}$ corresponding to the number of particles detected within the exposure time interval. Since the sources are isotropic point sources, the measured intensity only depends on the distances $d_{\mathbf{p}_{s_{i}} \mathbf{q}_{o_{j}}}$, between the position of observation $\mathbf{q}_{o_{j}}$ and the position of each source $\mathbf{p}_{s_{i}}$, and the background radiation intensity $\beta_{o_{j}}$, as expressed by (1) and (2). In particular, (2) describes the radiation field created by source $s_{i}$ for all space by combining the inverse square law model with an exponential absorption model (to describe the absorption of radiation particles in the air).

$$
\begin{gathered}
c_{o_{j}}=\sum_{i=1}^{n} f_{s_{i} o_{j}}+\beta_{o_{j}} \\
f_{s_{i} o_{j}}=s_{i} \frac{\mathrm{e}^{-\alpha d_{s_{i} o_{j}}}}{d_{s_{i} o_{j}}^{2}}
\end{gathered}
$$

The problem is to estimate the number of sources $N$ and the parameters (position and activity) of each source $\hat{\mathbf{s}}_{l}=\left(\mathbf{p}_{\hat{\mathbf{s}}_{l}}, a_{\hat{\mathbf{s}}_{l}}\right), l=1,2, \ldots, N$, given a set of observations.

\section{PROPOSED APPROACH}

Several approaches have been proposed to solve the problem described in Section II. In this section we will describe a number of them, and justify the selection of the approaches which will be implemented and tested in Section IV.

\section{A. Studied approaches}

1) Maximum Likelihood approach: The Maximum Likelihood approach (MLA) is based on the estimation of the source parameters which have the maximum likelihood for explaining the observations. The likelihood is calculated through the joint probability distribution of $N$ Poisson distributions (one for

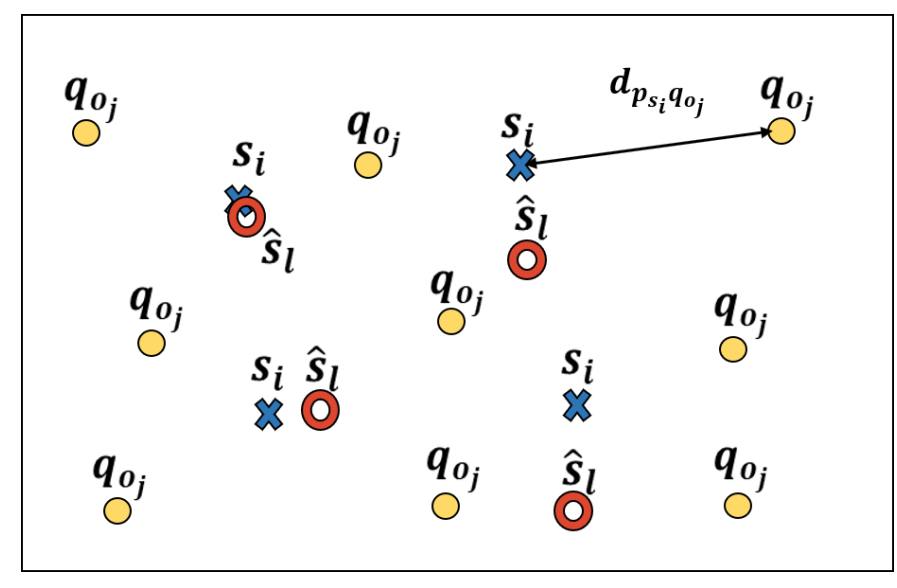

Fig. 1. Schematic representation of sources, observations, and estimated sources, illustrating the nomenclature used.

each potential source), where the samples correspond to the observed radiological intensity, and the distribution parameters are the positions and activities of the sources. Collecting the source parameters into a stacked vector $\mathbf{s}=\left[\mathbf{s}_{1}^{\top} \ldots \mathbf{s}_{N}^{\top}\right]^{\top}$, as well as the observations $\mathbf{o}=\left[\begin{array}{lll}o_{1} & \ldots & o_{m}\end{array}\right]^{\top}$, the maximum likelihood estimator is obtained from (3), where $p(\mathbf{o} \mid \mathbf{s})$ is the likelihood function.

$$
\hat{\mathbf{s}}=\arg \max _{\mathbf{s}} \log p(\mathbf{o} \mid \mathbf{s})
$$

In the work described in [15], this approach is applied to problems containing a single source. The authors use a small number of sensor measurements to localize and quantify the unknown source in a computational simulation. However, real world scenarios may have more than one source, so it is also necessary to determine the number of sources, in addition to estimating the parameters of each source.

In the work described in [16] the maximum likelihood approach is used to estimate the position and activity of an unknown number of sources. This work assumes that the radioactive sources are independent from each other and uses the sum of the contributions of each source in a computational simulation. However, this does not solve the problem that the number of sources remain unknown a priori. The proposed solution is to estimate the source parameters using the maximum likelihood for a different number of sources and then choose the solution with the highest score. The score of each solution is computed using (4), where $\hat{\mathbf{s}}_{\mathrm{ML}, r}$ is the maximum likelihood estimated source parameter vector, $\mathbf{J}$ is the Fisher information matrix, and $r$ is the number of sources being tested.

$$
\beta_{r}=\log p\left(\mathbf{o} \mid \hat{\mathbf{s}}_{\mathrm{ML}, r}\right)-\frac{1}{2} \log \left|\mathbf{J}\left(\hat{\mathbf{s}}_{\mathrm{ML}, r}\right)\right|
$$

2) Bayesian approach: This approach uses an initial guess for the source parameters which, through an iterative process, is updated until a convergence threshold is met or the limit of the number of iterations is reached.

In the work described in [16], the authors apply importance sampling to the Bayesian approach, drawing samples (particles) of the parameter vector and calculating their importance weight to approximate the integral in (5). In each iteration, 
particles are re-sampled according to their weight, which is proportional to the likelihood of that sample, given the observations which were made. Subsequently, each particle is updated according to a kernel density function.

$$
\hat{\mathbf{s}}_{\mathrm{B}}=\mathbb{E}[\mathbf{s} \mid \mathbf{o}]=\int \mathbf{s} \pi(\mathbf{s}) d \mathbf{s}
$$

After repeating this process a fixed number of times, the estimated parameter vector is taken to be the weighted average of all the particles. Similarly to what was described in the maximum likelihood approach, the number of sources is determined by repeating these steps for different numbers of sources, and choosing the solution which has the highest score. In this case, the score is given by the Bayesian information criterion, also known as the Schwartz criterion.

3) Robust Localization with Clustering approach: The Robust Localization with Clustering approach (RLCA) is based on a Monte Carlo technique which uses a set of particles and is centered around the idea of updating the set of particles biased towards the best-classified particles.

In the works described in [17] and [18], a multi-layer sequential Monte Carlo method coupled with suitable clustering algorithms is used to estimate the number, activities, and positions of sources. Each particle is a three-value vector representing a hypothetical source, containing the source's position (in $\mathbb{R}^{2}$ ) and activity. The initial set of particles is sampled from a uniform distribution. At each iteration of the recursive Bayesian estimation, the particles are classified by their weight, which is proportional to their respective likelihood given the observations. The particles with the lowest weight are replaced by particles with higher weights, and some are replaced with new randomly generated particles. In addition to the resampling process, a clustering process is applied to the particle set. The clustering process attempts to resolve the sources by estimating their parameters with the cluster centroid. The authors use three different clustering methods: mean-shift clustering, hierarchical clustering, and ID-based clustering. These two steps (resampling and clustering) are repeated until the confidence score is larger than a predefined threshold value.

4) Least Square Error approach: The Least Square Error approach (LSEA) attempts to fit a model to the data while minimizing the sum of square errors, as expressed by (6). In the work described in [19], the LSEA is used to estimate the position of one source using four sensors. The proposed solution is also able to track the position of the source over time. Once again, to determine the number of sources, (6) is solved for an increasing (but limited) number of sources, and the solution which produces the smallest sum of square errors is selected as the best estimator.

$$
\hat{\mathbf{s}}=\arg \min _{\mathbf{s}} \sum_{j=1}^{m}\left(c_{o_{j}}-\sum_{l=1}^{N} f\left(\mathbf{s}_{l}, \mathbf{q}_{o_{j}}\right)\right)^{2}
$$

\section{B. Selected approaches}

Due to differences in implementation complexity, not all of the approaches described in Subsection III-A were applied to test scenarios. The selected approaches are:

- MLA: it is simple to apply, can be used to identify the number of sources and their parameters, it is simple to adapt to the collimator model and it works with smaller numbers of observations, compared with other approaches.

- RLCA: it was tested in 3D simulations, it does not require repeating the estimation process for different numbers of sources, since it estimates the number of sources directly, and it is simple to adapt to the collimator model.

- LSEA: it produces good results with few observations in scenarios with one source, it requires fewer observations than other approaches in general, and it is simple to adapt to the collimator model.

\section{RESUlts}

In this section the experimental results are presented and discussed. Presented first are the results obtained in simulation, which, due to their quality, reduced the viable approaches to MLA and LSEA. Then, experimental results with real sources and sensors are presented, including the analysis of the application of a collimator.

The scenarios of experimentation are depicted in Figure 2 and explained as follows. The radiation sources are assumed to be static and located on the ground. The 2D experiments are associated to a human operator walking in the scenario, equipped with a mobile phone connected to the GMC, and with an approximately constant distance between the sensor and the ground. The $3 \mathrm{D}$ experiments are associated to the ones performed by UAV flying over the scenario at different altitudes, but assuming that each mission is performed with an approximately constant altitude, i.e., also a constant distance between the sensor and the ground. The 2D and 3D experiments are performed in simulated and real scenarios, as presented in the following subsections.

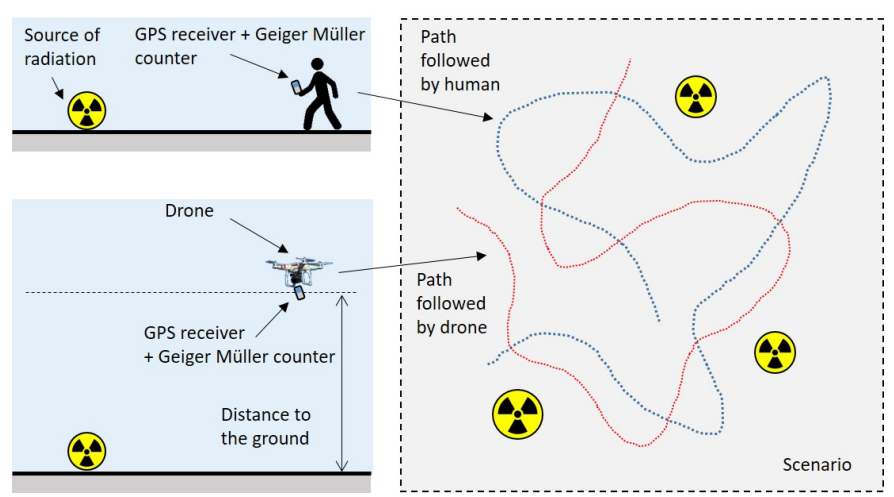

Fig. 2. $2 \mathrm{D}$ and $3 \mathrm{D}$ experiments associated to a human walking and a drone flying over the scenario, respectively (left images). The scenario, the sources of radiation and the described paths (right image). 


\section{A. Simulated Results}

A simulation environment was developed for the initial testing of each approach. In this environment, each source has a position in $\mathbb{R}^{3}$. The results compare the number of estimated sources with the number of simulated sources, and each test scenario was simulated several times to calculate a mean and a variance. Figure 3 shows an example graph to explain the axes and error bars. Under ideal conditions we would expect to see a linear relationship with the number of estimated sources equal to the number of simulated sources (i.e., the slope of the curve would be equal to 1 ).
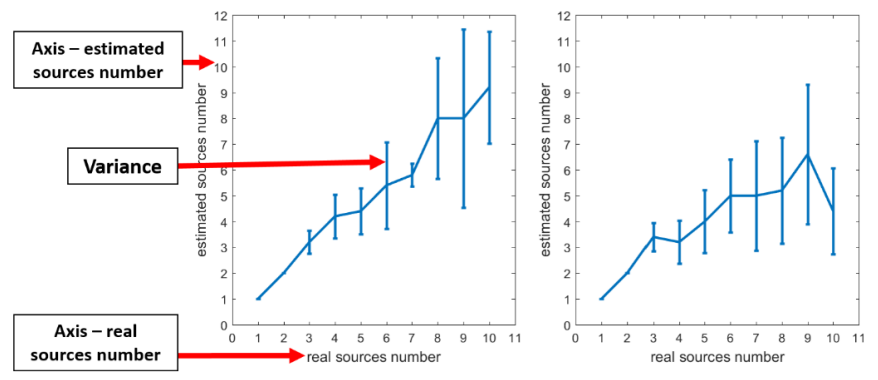

Fig. 3. Explanation of the elements of the result graphs. The horizontal axis represents the number of simulated sources and the vertical axis represents the number of estimated sources. Each simulation run has a fixed number of sources between 1 and 10 and is repeated several times to calculate the mean and the variance of the number of estimated sources.

We will start by comparing the results obtained when making observations in a grid pattern and the results obtained when making observations in random positions. The top image of Figure 4 shows observations made in a grid pattern in a scenario containing 3 sources, and the bottom image shows observations made in random positions in the same scenario. Illustrated in Figure 5 are the results of the estimation of the number of sources for MLA, RLCA and LSEA. It can be seen immediately that the RLCA produces poor results both in the case of grid positions and in the case of random positions. The MLA and LSEA produce similar results, with the MLA estimates having a smaller variance in the case of random positions. Due to the poor performance of the RLCA, it was abandoned in future tests.

Simulation results were also obtained for measurements made in 3 planes at $z=50 \mathrm{~cm}, z=60 \mathrm{~cm}$, and $z=70$ $\mathrm{cm}$. The results for the estimated number of sources in this scenario are presented in Figure 6. Here we can see that the MLA performs better than the LSEA, especially for a greater number of sources.

Following the results of Figures 5 and 6, all subsequent tests were performed using only the MLA, since this approach produced the best results.

Up to this point no uncertainty was introduced in the observations so they were taken as perfect observations, both in regards to the position $\mathbf{q}_{o_{j}}$ and in regards to the measured intensity $c_{O_{j}}$. Thus, in order to test the robustness of the Maximum Likelihood Approach, tests were made with noise in the measured intensity, with noise in the observation position, and with noise in both. The results are presented in Figure 7. It can be seen that the results deteriorate considerably,

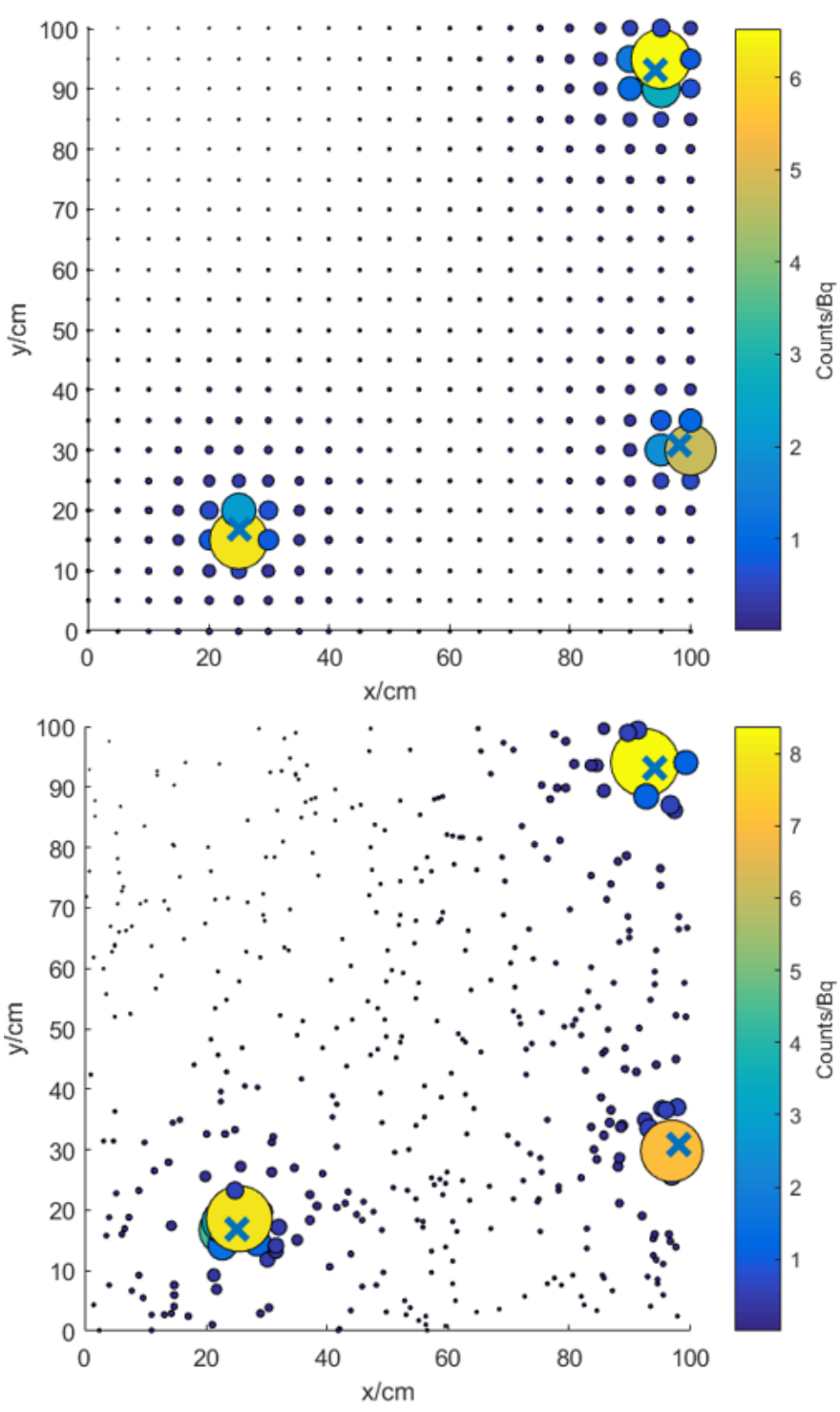

Fig. 4. Simulation of observations in a grid pattern and in random positions (top and bottom images, respectively). The blue crosses represent the source positions and the color and radius of the circles represent the measured intensity.

especially when there is noise in the observation position. In general, except for the case where there is only one source being simulated, the MLA greatly underestimates the number of sources in the scenario. The most striking example is the case of observations made in a grid pattern with noise in position and measured intensity, where the MLA estimates 1.5 sources on average when there are between 1 and 4 sources being simulated, and approximately 2.5 sources on average when there are 5 sources being simulated. We conclude then that the MLA is quite sensitive to noise in the observations, and that, in general, it underestimates the number of sources.

1) Application of a collimator: One way to improve the results observed in Figure 7 is with the application of a collimator. A collimator gives an effective orientation to the simulated measurements by only taking into consideration the sources which are inside a cone defined by the collimator angle $\omega$. Figure 8 illustrates the collimator's effect. 

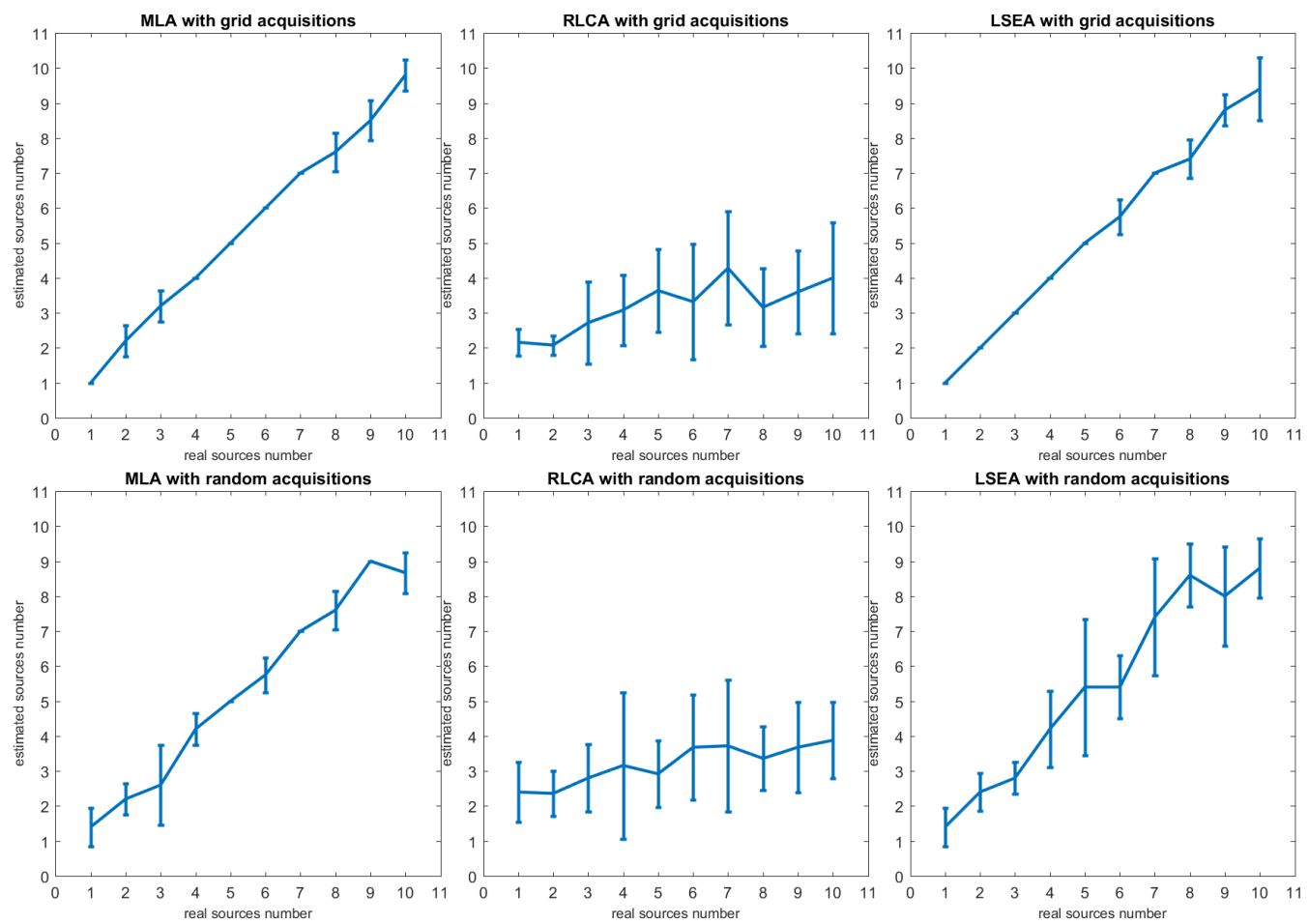

Fig. 5. Simulation results with measurements made at a height of $0 \mathrm{~cm}$. The top row refers to measurements made in a square grid pattern. The bottom row refers to measurements made in random positions. In all figures the horizontal axis is the real number of sources, and the vertical axis is the estimated number of sources.
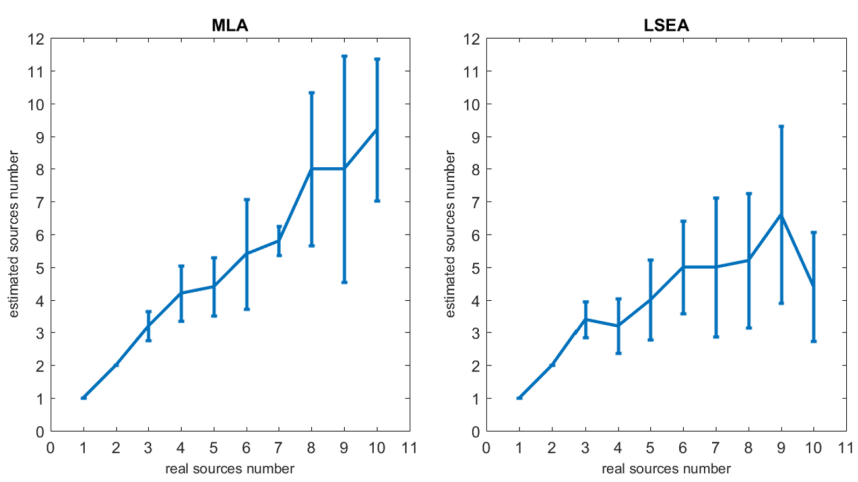

Fig. 6. Simulation results with measurements made in a square grid pattern at heights of 50,60, and $70 \mathrm{~cm}$. Left: results for the Maximum Likelihood Approach. Right: results for the Least Square Errors Approach.

Thus, by simulating the effect of a collimator with $\omega=15^{\circ}$ the MLA results are improved, as shown in Figure 9. It can be seen that the estimated number of sources is greatly improved when compared to the results in Figure 7, especially in the case of observations with position noise (third column from the left). However, in the case of noise both in position and in measured intensity, results are still poor and generally have a large variance.

\section{B. Experimental Results}

In order to further validate the application of the MLA to radiological source detection and characterization, tests were also performed in laboratory conditions and in a real-world
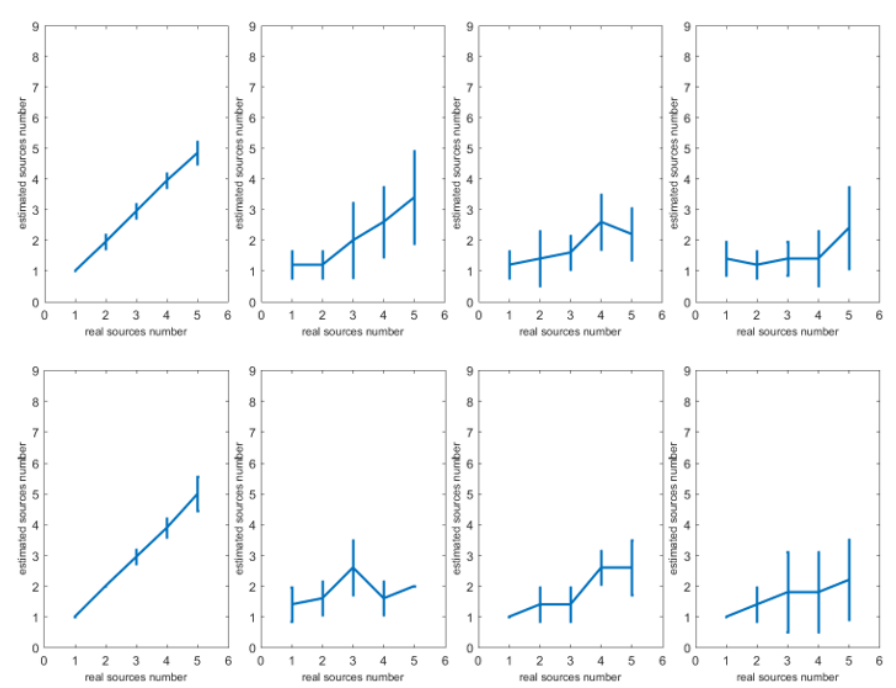

Fig. 7. Effect of intensity and position noise on the estimation of the number of sources. All noise levels are 20\%. Top row: grid pattern. Bottom row: random positions. Columns from left to right: no noise, intensity noise, position noise, intensity and position noise.

scenario containing radioactive sources. Additionally, a lead collimator was constructed to assess the potential improvement in source characterization, since the GMC is approximately cylindrical and, without a collimator, the measured intensity depends almost exclusively on the distance to the sources.

The collimator which was built for testing is shown in Figure 10. The collimator is made out of lead (which has a large absorption coefficient) and has the shape of a satellite 


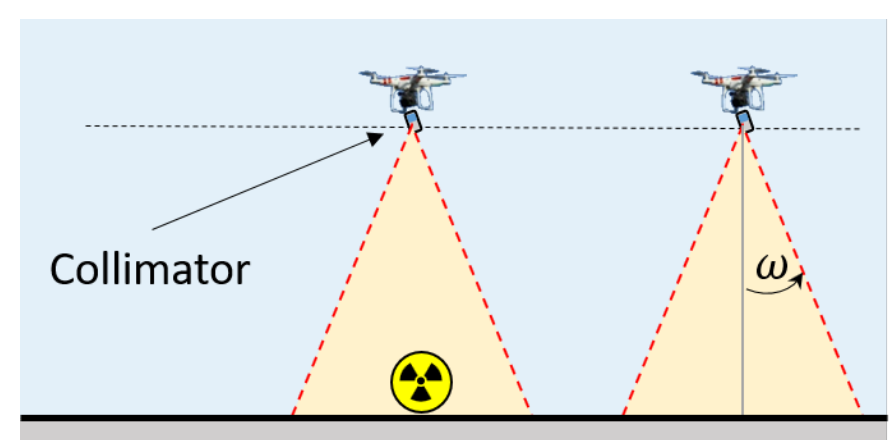

Fig. 8. Schematic representation of the collimator's effect. Only the sources inside the cone defined by $\omega$ contribute to the intensity measured.
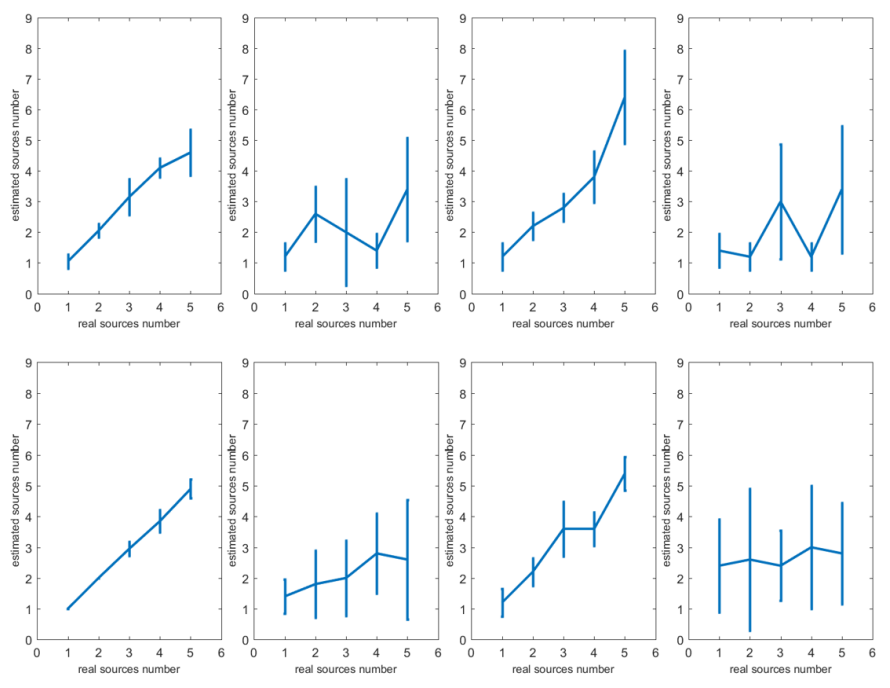

Fig. 9. Application of a collimator: effect of intensity and position noise on the estimation of the number of sources. Observations are simulated in the plane $z=5 \mathrm{~cm}$ and the collimator has an angle $\omega=15^{\circ}$. All noise levels are $20 \%$. Top row: grid pattern. Bottom row: random positions. Columns from left to right: no noise, intensity noise, position noise, intensity and position noise.

dish. The hole in the center allows radiation particles to reach the detector. However, since the collimator only covers the "front" of the Geiger-Müller tube, particles can also be detected from the sides. The measured intensity as a function of the GM tube's orientation towards the source is shown in Figure 11. This figure compares the intensity vs angle curves with and without the collimator. It can be seen that the collimator has the desired effect for angles smaller than $60^{\circ}$, allowing radiation to be detected for very small angles and then absorbing radiation for larger angles. But, for angles larger than $60^{\circ}$ the collimator's effect starts to become negligible.

Experiments were then made to test whether the collimator improved the results of the MLA using a real radioactive source in a laboratory environment. Measurements were made with a GMC in a grid pattern in two scenarios: with and without the collimator. The results are illustrated in Figure 12. These results confirm that the collimator improves the localization of the source, although this improvement comes at a price: the measured intensity is an order of magnitude smaller, which decreases the signal-to-noise ratio, i.e., the

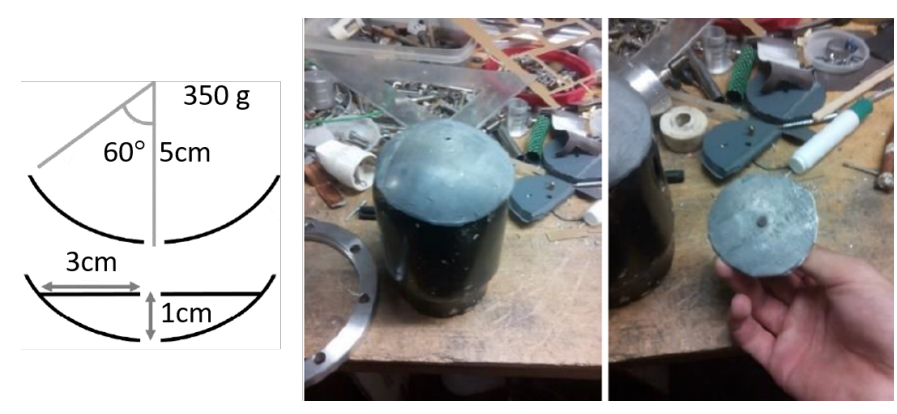

Fig. 10. Left: collimator design. Right: photographs of the collimator which was built for testing.

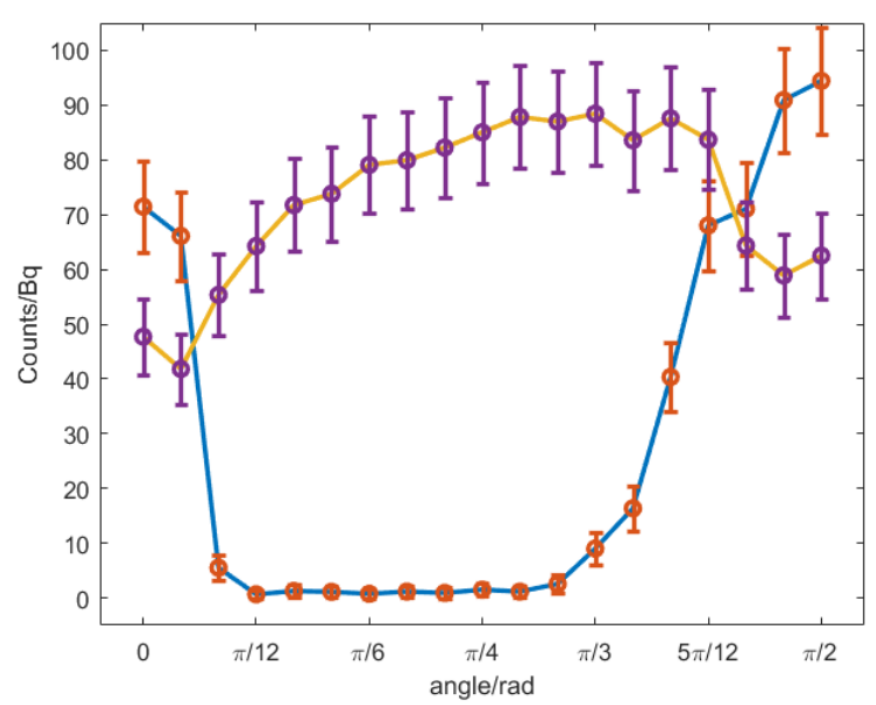

Fig. 11. Comparison between sensor readings with (blue/orange) and without (yellow/purple) a collimator as a function of the orientation towards the source. Due to the "satellite dish" shape of the collimator, intensity readings begin to increase above $60^{\circ}$ since the sensor is not shielded on its sides.

measurements become more sensitive to noise. Nonetheless, we can conclude that the collimator does increase the accuracy of the source's estimated location.

Finally, experiments were made in a real-world setting containing two radioactive sources. The GMC was connected to a smartphone for recording and georeferencing the measurements, and two situations were tested: first, a human operator carried the sensor and smartphone and walked around the scenario (Figure 13, left); second the sensor and smartphone were mounted on a UAV (Figure 13, right). Due to the limited payload of the UAV, it was not possible to include the collimator. The radioactive sources were placed on the ground in a courtyard at IST, as shown in Figure 14. One test was performed with a human operator and two tests were performed with the UAV, represented in Figure 15.

\section{Conclusions}

Three approaches for quantification of radiological sources were tested: robust localization with clustering (RLCA), least square error (LSEA), and maximum likelihood (MLA). The MLA provided the best results when considering 2D and 3D scenarios, and was therefore selected for further testing. It was 

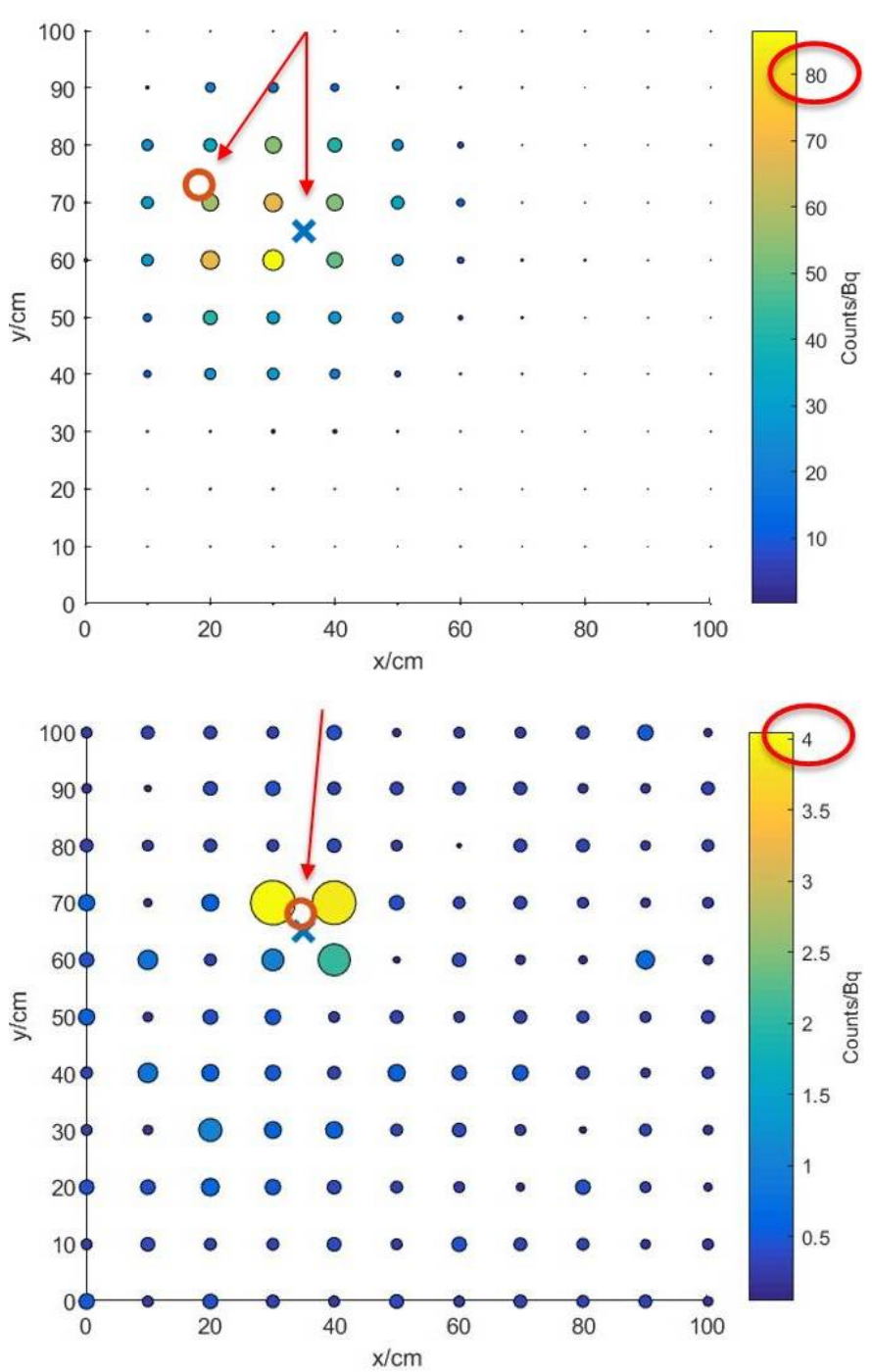

Fig. 12. Comparison of the estimated map without and with a collimator (top and bottom images, respectively).

shown that a collimator provides considerable improvement in the localization of sources, but significantly reduces the payload of a UAV and also decreases the signal-to-noise ratio. For future work the authors will perform new experiments with a more efficient Geiger-Müller counter (namely, the Mazur PRM-9000), and will develop new research into path planning and area coverage to maximize information gain and reduce mission length.

\section{ACKNOWLEDGMENT}

This paper is a result of the project the project FRIENDS Fleet of dRones for radIological inspEction, commuNication anD reScue, [20] supported by the "Fundação para a Ciência e Tecnologia" (FCT), Compete 2020 and Lisboa 2020 under the PORTUGAL 2020 Partnership Agreement, through the European Regional Development Fund (ERDF). IPFN activities also received financial support from FCT though project UID/FIS/50010/2019.
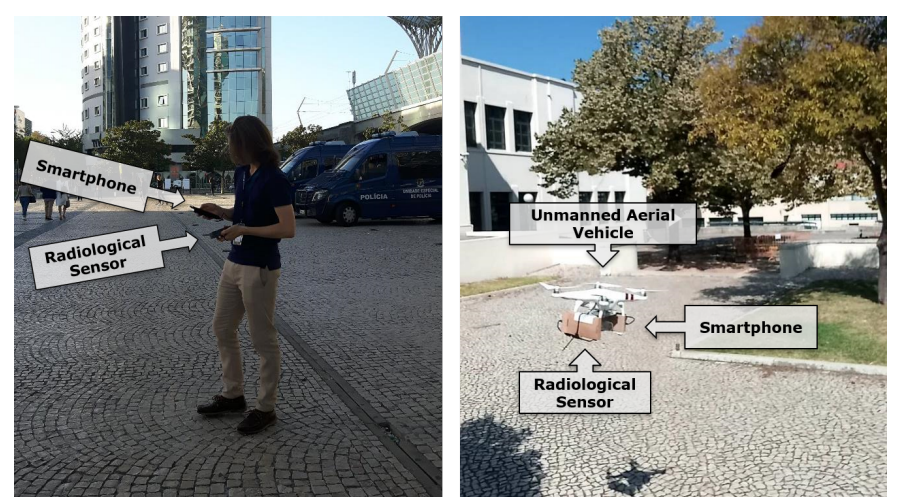

Fig. 13. Left: a human operator carrying the radiological sensor connected to a smartphone. Right: a UAV mounted with the radiological sensor connected to a smartphone.

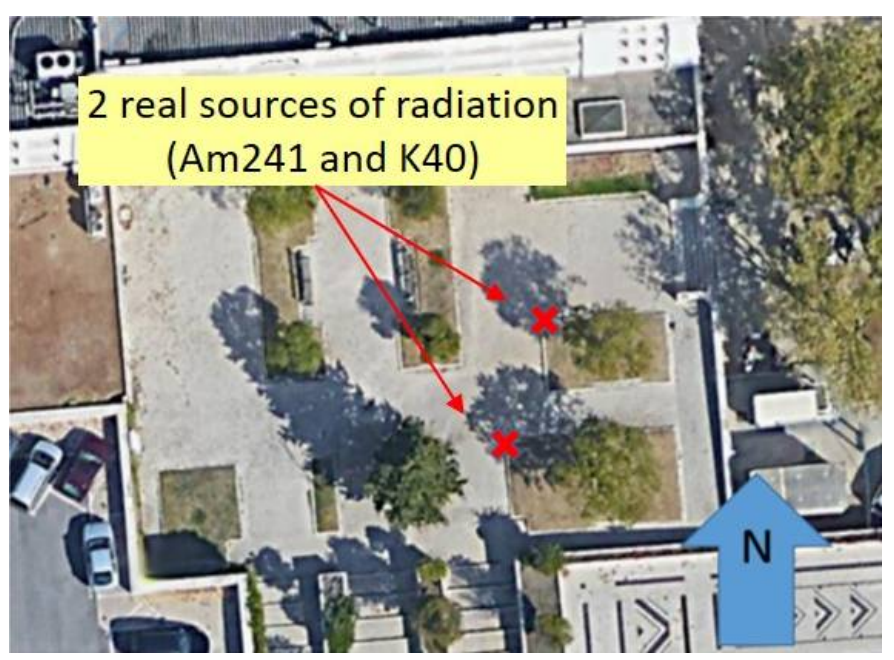

Fig. 14. Map view illustrating the source positions and the origin of the reference frame (yellow circle). Map provided by Google Maps ${ }^{\mathrm{TM}}$.

\section{REFERENCES}

[1] J. Chamot and R. Atkins. (2008, February) Leading engineers and scientists identify advances that could improve quality of life around the world. National Science Foundation. [Online]. Available: https://www.nsf.gov/news/news_summ.jsp?cntn_id=111158

[2] National Academy of Engineering, "NAE Grand Challenges for Engineering," 2008. [Online]. Available: https://www.nae.edu/187212/ NAE-Grand-Challenges-for-Engineering

[3] — Grand Challenges - Prevent Nuclear Terror. National Academy of Engineering. [Online]. Available: http://www.engineeringchallenges. org/challenges/nuclear.aspx

[4] Orano DS, "Press Release - Dismantling Robots 2018," 2018. [Online]. Available: https://www.orano.group/en/press/dossiers-de-presse/robotsdismantling-and-services

[5] M. Lozano and A. Fernandez. Reward Project. [Online]. Available: http://www.reward-project.eu/

[6] Academia da Força Aérea. (2015) Project seagull. [Online]. Available: http://www.academiafa.edu.pt/subPagina-10D00-019. 005.003.011-seagull

[7] Framatome. (2016) WNE: AREVA Awarded in the Fields of Safety and Innovation. [Online]. Available: http://www.framatome.com/EN/businessnews-211/wne-arevaawarded-in-the-fields-of-safety-and-innovation.html

[8] ISQ. (2015) Gamma-Ex. [Online]. Available: https://www.isq.pt/projeto/ gamma-ex/

[9] ENCIRCLE Consortium. ENCIRCLE: European CBRN Innovation for the Market Cluster. European Union. [Online]. Available: http://encircle-cbrn.eu/ 
[10] C. Cai, B. Carter, M. Srivastava, J. Tsung, J. Vahedi-Faridi, and C. Wiley, "Designing a radiation sensing uav system," in 2016 IEEE Systems and Information Engineering Design Symposium (SIEDS). IEEE, 2016. [Online]. Available: http://ieeexplore.ieee.org/document/7489292/

[11] K. Boudergui, F. Carrel, T. Domenech, N. Guénard, J.-P. Poli, A. Ravet, V. Schoepff, and R. Woo, "Development of a drone equipped with optimized sensors for nuclear and radiological risk characterization," in 2011 2nd International Conference on Advancements in Nuclear Instrumentation, Measurement Methods and their Applications. IEEE 2011. [Online]. Available: http://ieeexplore.ieee.org/document/6172936/

[12] Mirion Technologies. SPIR-Explorer Sensor. [Online]. Available: https://www.mirion.com/products/spir-explorer-sensor-light-widerange-radiological-detection-and-identification-sensor

[13] FlyCam UAV. Drone UAV Aerial Radiation Detection. [Online]. Available: https://www.flycamuav.com/aerial-radiation-detection/

[14] H. Carvalho, A. Vale, R. Marques, R. Ventura, Y. Brouwer, and B. Gonçalves, "Remote inspection with multi-copters, radiological sensors and slam techniques," EPJ Web of Conferences, vol. 170, 2018. [Online]. Available: https://www.epj-conferences.org/10.1051/epjconf/ 201817007014

[15] H. E. Baidoo-Williams, "Maximum likelihood localization of radiation sources with unknown source intensity," ArXiv e-prints, 2016. [Online]. Available: http://arxiv.org/abs/1608.00427

[16] M. Morelande, B. Ristic, and A. Gunatilaka, "Detection and parameter estimation of multiple radioactive sources," in 2007 10th International Conference on Information Fusion. IEEE, 2007. [Online]. Available: http://ieeexplore.ieee.org/document/4408094/

[17] J. Chin, D. K. Y. Yau, and N. S. V. Rao, "Efficient and robust localization of multiple radiation sources in complex environments," in 201131 st International Conference on Distributed Computing Systems. IEEE, 2011. [Online]. Available: http://ieeexplore.ieee.org/document/5961730/

[18] D. Shah and S. Scherer, "Robust localization of an arbitrary distribution of radioactive sources for aerial inspection," Computing Research Repository, 2017. [Online]. Available: http://arxiv.org/abs/1710.01701

[19] J. W. Howse, L. O. Ticknor, and K. R. Muske, "Least squares estimation techniques for position tracking of radioactive sources," Automatica, vol. 37, no. 11, 2001. [Online]. Available: https: //www.sciencedirect.com/science/article/pii/S0005109801001340

[20] IPFN. (2018) FRIENDS: Fleet of drones for radiological inspection, communication and rescue. Instituto Superior Técnico. [Online]. Available: https://www.ipfn.tecnico.ulisboa.pt/FRIENDS/
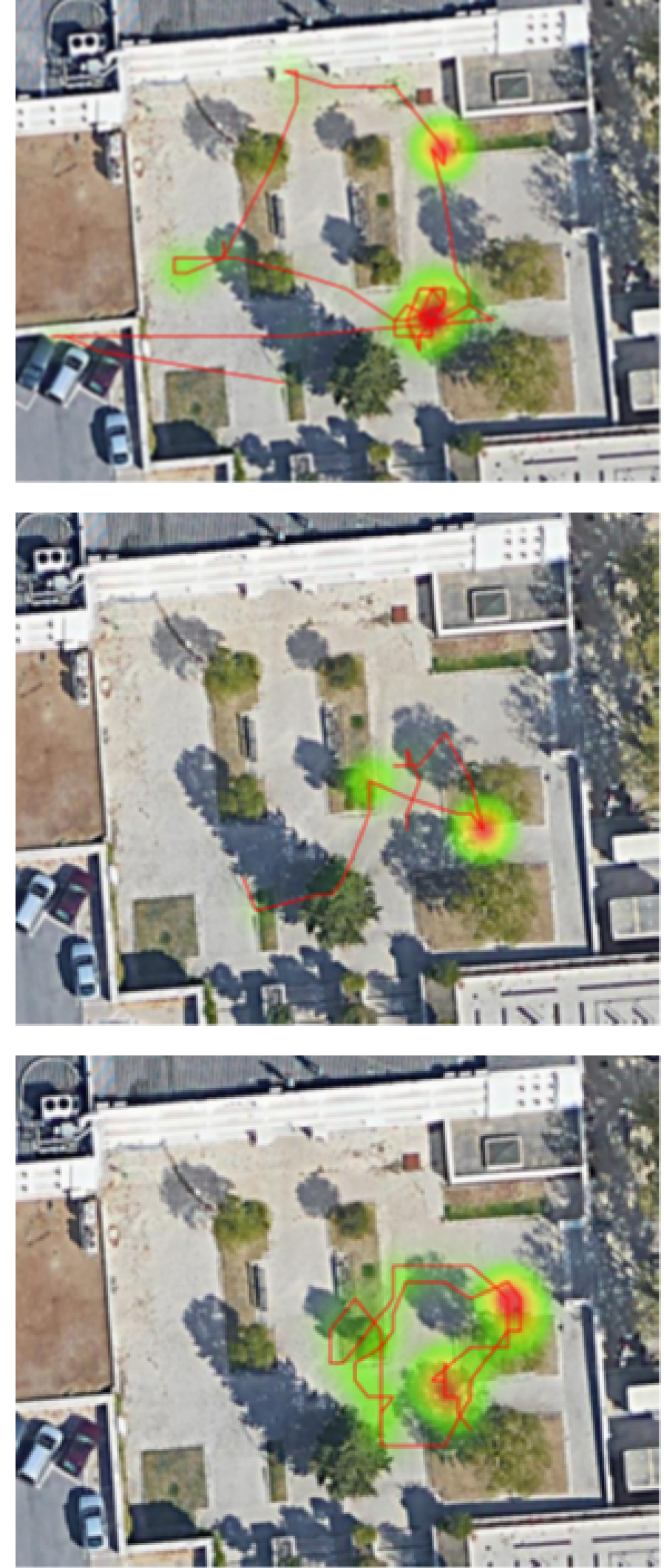

Fig. 15. Experimental tests in a controlled scenario of IST campus with two sources of radiation. From top to down: walking in the scenario with the sensor on hand and two tests with a UAV flying over the scenario. 